\title{
Hydatid cyst mimicking cystic neoplasm of the pancreatic tail: an endoscopic ultrasound diagnosis
}

A 59-year-old woman presented with weight loss and left hypochondrial discomfort that had been present for 2 weeks. Abdominal computed tomography (CT) revealed a $12 \mathrm{~cm} \times 11 \mathrm{~cm}$ lobulated nonenhancing cystic lesion with intrinsic heterogeneous hyperdense areas ( $\bullet$ Fig.1) and peripheral wall calcification ( Fig.1b). A provisional diagnosis of cystic neoplasm of the tail of the pancreas was made and the patient was referred for endoscopic ultrasound (EUS) and fine needle aspiration (FNA).

EUS (3870UTK; Pentax Tokyo, Japan) revealed a cystic lesion adjacent to the pancreatic tail and continuous with the spleen ( Fig. 2 a). The EUS features were characteristic for an hydatid cyst. Multiple serpentine structures ( $\mathbf{F i g . 2 b}$ ) representing collapsed membranes were seen within the cyst. A daughter cyst ( $\bullet$ Fig. 2c) containing echogenic material was noted, and also interspersed calcifications were observed inside the cyst cavity ( $\bullet$ Fig. 2 d). The echogenic material seen inside the cyst (akin to gallbladder sludge) is termed "hydatid sand" and it settles at the most dependent portion of the cyst. FNA of cyst fluid was not done because of the risk of anaphylaxis from spillage or leak of cystic fluid during FNA [1]. An enzyme-linked immunosorbent assay (ELISA) for Echinococcus IgG antibody was positive. The patient was referred for surgery, after initiation of treatment with albendazole at a dose of $15 \mathrm{mg} / \mathrm{kg}$ bodyweight in two divided daily doses (not to exceed $800 \mathrm{mg} /$ day) for 28 days.

Hydatid disease is a worldwide zoonosis caused by the Echinococcus tapeworm. Ultrasound features are characteristic although they may vary and several classifications based on the cyst appearance have

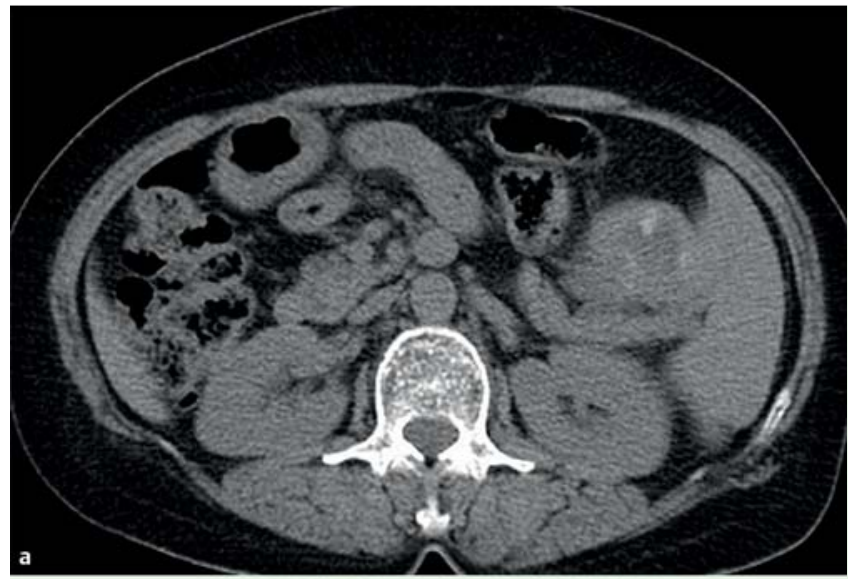

Fig. 1 Abdominal computed tomography (CT) revealed a lobulated nonenhancing cystic lesion with: a intrinsic heterogeneous hyperdense areas; $\mathbf{b}$ thick peripheral wall calcification.

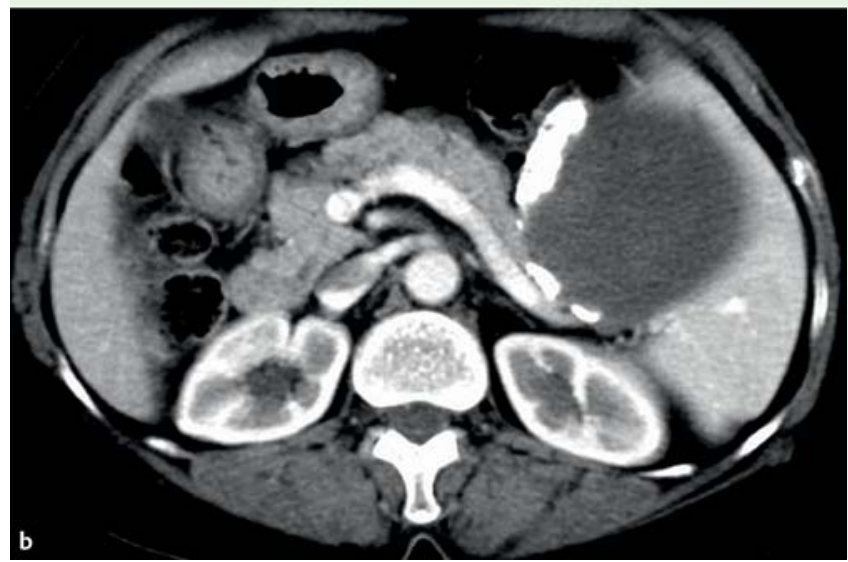

been proposed [2,3]. EUS (as in our patient) can be equally effective in providing high resolution images to demonstrate characteristic cyst features when other imaging modalities are unreliable or unavailable [4]. To the best of our knowledge, this is the first such demonstration in the published literature of EUS visualization of features of an unruptured hydatid cyst.

Endoscopy_UCTN_Code_CCL_1AF_2AZ_3AD

Competing interests: None
Suvadip Chatterjee', Manas K. Roy ${ }^{2}$, Sudeep Banerjee ${ }^{2}$, Sumit Mukhopadhaya $^{3}$, Saugata Sen ${ }^{3}$, Mohandas

\section{K. Mallath ${ }^{1}$}

${ }^{1}$ Department of Gastroenterology, Tata Medical Center, Kolkata, India

2 Department of GI Surgery, Tata Medical Center, Kolkata, India

${ }^{3}$ Department of Radiology, Tata Medical Center, Kolkata, India 

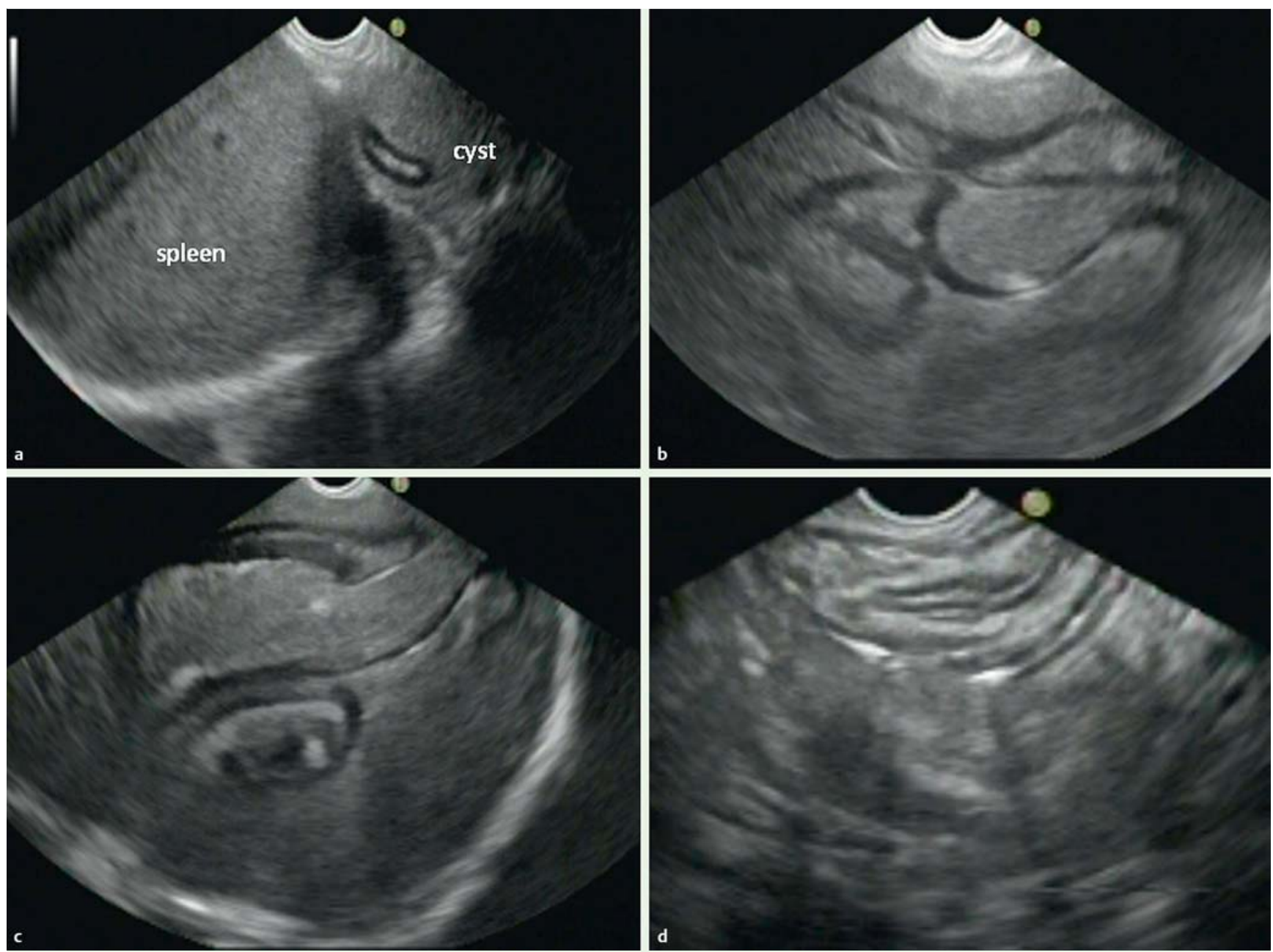

Fig. 2 Endoscopic ultrasound (EUS) appearances of a hydatid cyst. a A large cyst continuous with the spleen. $\mathbf{b}$ Multiple serpentine structures representing collapsed membranes were seen within the cyst cavity. $\mathbf{c}$ A daughter cyst with echogenic material. $\mathbf{d}$ Calcifications were also noted within the cyst cavity and on the cyst wall.

\section{References}

1 Neumayr A, Troia G, de Bernardis $C$ et al. Justified concern or exaggerated fear: the risk of anaphylaxis in percutaneous treatment of cystic echinococcosis - a systematic literature review. PLoS Negl Trop Dis 2011; 5: e1154

2 Lewall DB, McCorkell SJ. Hepatic echinococcal cyst: sonographic appearance and classification. Radiology 1985; 155: 773-775

3 Gharbi HA, Hassine W, Brauner MW et al. Ultrasound examination of the hydatic liver. Radiology 1981; 139: 459-463
4 Sharma M, Somasundaram A, Pathak A et al. Endoscopic ultrasound in hepatobiliary hydatid disease. Endoscopy 2010; 42 (Suppl. 02): E56-E57

\section{Bibliography}

Dol http://dx.doi.org/

10.1055/s-0034-1377187

Endoscopy 2014; 46: E318-E319

(c) Georg Thieme Verlag KC

Stuttgart · New York

ISSN 0013-726X

\section{Corresponding author}

\section{Suvadip Chatterjee, MD}

Tata Medical Center

14-16, Main Arterial Road

Rajarhat Newtown

Kolkata 700156

India

Fax: +91-33-66057587

suvadip_chatterjee@yahoo.com 